\title{
Toxina botulínica: relación entre tipo de paciente y duración del efecto
}

\section{Botulinum toxin: relation between patien type and duration of the effect}

\author{
De Maio, M. *, Ofenböck Magri, I.**, Narvaes Bello, C.***
}

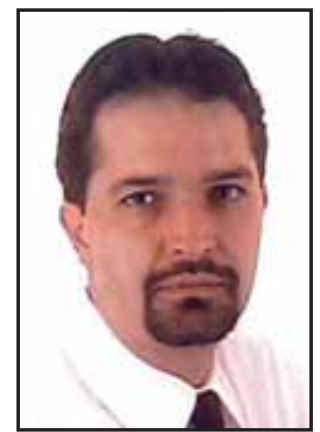

De Maio, M

\section{Resumen}

El uso de la Toxina Botulínica es frecuente en procedimientos de Cirugía Cosmética. Sin embargo, todavía quedan muchos aspectos que necesitan ser aclarados tales como los tipos de pacientes y la duración de su efecto. Si agrupamos los pacientes en diferentes modelos según su función muscular, podremos predecir la duración de ese efecto e informar adecuadamente a los pacientes. Estos modelos los denominamos cinético, hipercinético y hipertónico.

El modelo cinético es el de aquellos pacientes que presentan una concordancia entre su expresividad facial y sus emociones y que muy probablemente solo necesitarán un tratamiento al año. El modelo hipercinético incluye a aquellos pacientes que no mueven sus músculos de acuerdo con su mímica; sus músculos faciales se contraen más rápidamente. En este caso la duración del tratamiento variará de los 4 a los 6 meses. El modelo hipertónico lo encontramos en aquellos pacientes que son incapaces de relajar sus músculos faciales, en los que la duración del tratamiento solo será de 2 a 3 meses. Esto es lo que puede hacer que en estos casos el tratamiento defraude tanto al paciente como al médico.

Mediante esta comprensión del comportamiento muscular, cada paciente debe ser encajado en su grupo correspondiente de acuerdo a sus características musculares, lo que nos permitirá predecir la duración del efecto y guiar futuros tratamientos.

\section{Palabras clave Toxina Botulínica, Cirugía cosmética, modelos musculares.}

Código numérico 263, 266
The use of Botulinum Toxin is frequent in non surgical cosmetic procedures. Many aspects, though, still need clarification such as patient types and the duration of effect. By grouping the patients in different muscle pattern, the duration can be predicted and informed to patients. These groups consist of kinetic, hyperkinetic and hypertonic patterns.

The kinetic pattern indicates that patients present a concordance with facial expression and emotions and they will probably need only one treatment per year. The hyperkinetic pattern refers to patients who do not move their muscles according to their mimicry. Facial muscles contract faster and the duration of the treatment will vary from 4 to 6 months. The hypertonic pattern is found in those patients that are unable to relax their facial muscles and the duration may be only from 2 to 3 months. That makes the treatment disappointing both for patients and injectors.

By understanding the muscle behavior, the patient approach and their grouping according to specific muscle patterns, enables the prediction of duration and guidelines to future treatments.

$\begin{array}{ll}\text { Key words } & \begin{array}{l}\text { Botulinum Toxin, Cosmetic surgery, } \\ \text { Muscle pattern }\end{array} \\ \text { Numeral Gode } & 263,266\end{array}$




\section{Introducción}

El tratamiento estético con Toxina Botulínica continua siendo una de las técnicas no quirúrgicas más frecuentemente empleadas entre todos los procedimientos mínimamente invasivos de Cirugía Cosmética. Diferentes técnicas y tácticas siguen siendo descubiertas e implantadas para el tratamiento de los tercios superior, medio e inferior de la cara. A pesar del avance del conocimiento médico, quedan todavía algunas cuestiones para las que solo tenemos respuestas insatisfactorias. Una de estas cuestiones es el tiempo de duración del efecto de la Toxina Botulínica y el porqué existe una variación tan significativa del mismo entre distintos pacientes (1).

Este trabajo intenta aportar datos que faciliten el abordaje de los pacientes haciendo posible la inclusión de los mismos en diferentes grupos según su comportamiento muscular, así como informar y orientar en relación a la duración del efecto a conseguir en tratamientos futuros $(2,3)$.

\section{COMPORTAMIENTO MUSCULAR}

El comportamiento de los músculos se altera durante el proceso de envejecimiento. La disminución de la agudeza visual, el exceso de piel en los párpados superiores y la ptosis de las cejas favorecen la contracción exagerada de los corrugadores y del músculo frontal respectivamente.

La musculatura de la mímica, además de expresar emociones, puede ser útil en los procesos compensatorios y de adaptación que se producen cuando envejecemos (4). Los músculos elevadores de la cara se vuelven progresivamente más débiles y los depresores, por la disminución de la fuerza antagónica, comienzan a contraerse favoreciendo el aspecto de cansancio y de tristeza, como sucede con el músculo depresor del ángulo de la boca $(5,6)$.

Cuando observamos a algunos pacientes mientras se expresan o conversan, notamos diferentes patrones de la mímica: los pacientes "normales", los que se expresan exageradamente y aquellos que se expresan de manera contenida (5).

\section{HISTORIA}

¿Quién no ha escuchado nunca de boca de un paciente que no quería quedar con la "cara espantada o paralizada" por usar Toxina Botulínica?. Inicialmente, algunos tratamientos se realizaban de forma excesiva, provocando un bloqueo muscular no deseado. Como resultado, además de la insatisfacción de los pacientes, se estigmatizó el uso de la Toxina Botulínica (7).
La paralización completa de algunos grupos musculares llevada a cabo en pacientes por motivos estéticos también era fuente de confusión para los pacientes y para algunos médicos. Un resultado se consideraba eficaz cuando producía un bloqueo muscular total, con ausencia de movimientos. Tras 28 días de la aplicación, se producía de nuevo el funcionamiento del axón terminal y el paso de acetilcolina, con lo que se iniciaba de nuevo el movimiento muscular, lo que para muchos pacientes conllevaba un concepto erróneo de finalización del efecto (8).

\section{TIPOS DE PACIENTES \\ Pacientes cinéticos}

Denominamos pacientes cinéticos a aquellos que mueven su musculatura facial de acuerdo con las emociones que desean transmitir. Cuando desean expresar sorpresa, elevan las cejas; el enfado se manifiesta mediante contracción de los corrugadores. La contracción y la relajación musculares presentan un ritmo agradable para el interlocutor, que se siente cómodo cuando conversa con este tipo de pacientes. La frase de referencia para este tipo de pacientes es "Yo contraigo la musculatura cuando expreso emociones" $(9,10)$. En el examen físico, las líneas y arrugas faciales aparecen solamente durante la contracción muscular y en general no son profundas. No hay presencia de líneas estáticas en las áreas de movimiento muscular y los músculos presentan un tono muscular normal.

La duración del efecto de la Toxina Botulínica en estos pacientes cinéticos es la mayor de las encontradas entre los diferentes tipos de pacientes. No es infrecuente que estos pacientes hagan una aplicación de Toxina botulínica una vez al año; no porque la

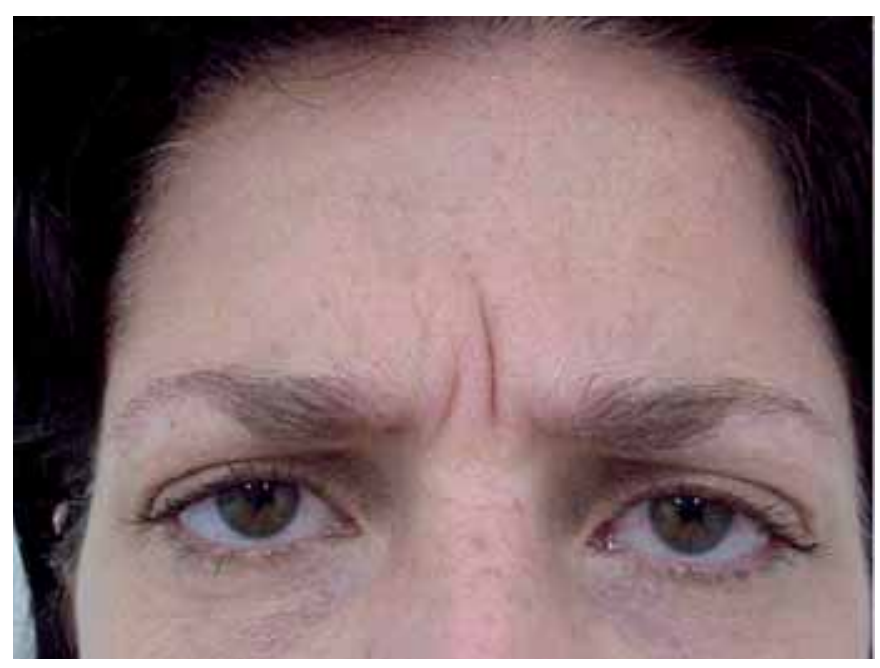

Fig. 1. Pacientes cinéticos: por lo general necesitan solamente una aplicación anual de Toxina Botulínica.

Pacientes hipercinéticos:necessitam em geral somente uma aplicação por ano. 


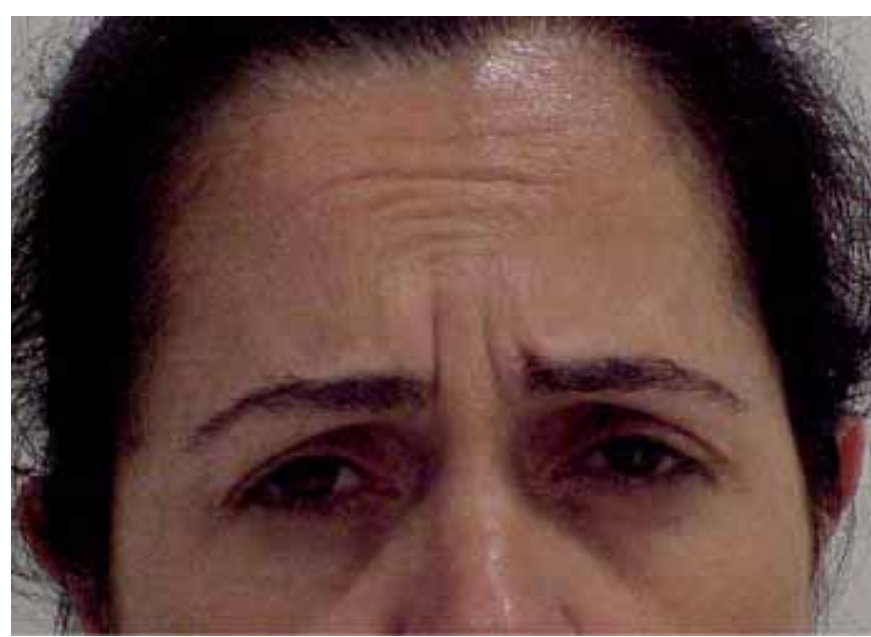

Fig. 2. Pacientes hipercinéticos: no presentan relación entre la contracción muscular y la expresión de una emoción. La duración del efecto de la Toxina Botulínica puede ser de 4 a 6 meses.

Pacientes hipercinéticos: não apresentam relação entre a contração muscular e a expressão da emoção. A duraçao de efeito é de 4 a 6 meses.

parálisis muscular dure un año, sino porque el movimiento muscular no incomoda al paciente hasta el punto de hacerle necesitar más de una inyección al año.

Para este tipo de pacientes es útil la información de que el efecto de la Toxina Botulínica puede durarles más de 6 meses, a veces, hasta 9 meses (Fig. 1).

\section{Pacientes hipercinéticos}

Son aquellos que contraen su musculatura facial a un ritmo más rápido y no necesariamente en concordancia con sus emociones. El interlocutor tiene la sensación de inquietud, pues observa excesivas contracciones de la musculatura mímica. Hay un ritmo acelerado en la alternancia de contracción y relajación. La frase de referencia para este tipo de pacientes es "No consigo controlar mi mímica".

En el examen físico, las líneas y arrugas faciales están presentes durante el reposo, si bien no son profundas. Durante la contracción muscular, notamos que la fuerza muscular produce la profundización intensa de estas líneas. En general, la líneas desaparecen si las aplastamos con los dedos y a la palpación, la dermis tiene un espesor uniforme y regular.

Para estos pacientes hipercinéticos, la duración del efecto de la Toxina Botulínica es conocida y establecida por la literatura: de 4 a 6 meses. Son los únicos en los que se puede prever fácilmente la duración del efecto. En general, si se aproxima a los 6 meses se sienten extremadamente felices. Si el efecto solo dura 4 meses, los pacientes acostumbran a quejarse, pero nosotros les habremos avisado previamente de esta posibilidad. Este tipo de pacientes vuelve con frecuencia para nuevas aplicaciones de Toxina Botulíni-

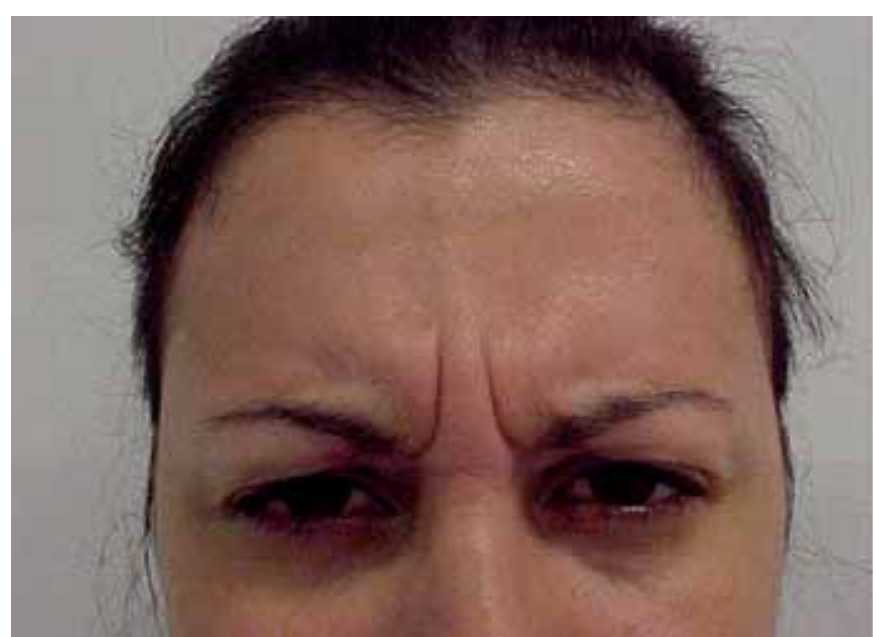

Fig. 3. Pacientes hipertónicos: presentan dificultad para relajar la musculatura facial. La duración del efecto de la Toxina Botulínica puede ser solamente de 2 a 3 meses.

Pacientes hipertônicos: presentam dificuldade em relaxar a musculatura facial. A duração de efeito pode ser de somente 2 a 3 meses.

ca, puesto que hay un grado alto de satisfacción con los resultados. La presencia de movimiento muscular les estimula a realizar tratamientos consecutivos (11) (Fig. 2).

\section{Pacientes hipertónicos}

La característica de los pacientes hipertónicos es su dificultad para relajar la musculatura facial. En general, áreas como la glabela, la región frontal y la comisura oral, presentan arrugas profundas tanto en reposo como en movimiento.

En el examen físico, cuando solicitamos al paciente que realice movimientos de contracción y de relajación de la musculatura de la mímica, notamos que es incapaz de lograr una relajación total; permanece siempre un grado variable de contracción. A la palpación notamos que hay irregularidades en la piel y una gran disminución de la dermis a lo largo de las arrugas.

Este grupo de pacientes es más complicado de abordar en lo que respecta a la duración del efecto de la Toxina Botulínica. Por su musculatura hipertónica, deben objetivar en las primeras aplicaciones un bloqueo muscular y la consecuente relajación de la región tratada, pero no la desaparición completa de la arruga. Por lo tanto, este tipo de pacientes debe ser advertido de que la duración del efecto en su caso puede ser de 2 a 3 meses. Está claro que esto puede no gustarles, pero es siempre mejor que el paciente sea siempre avisado previamente (12).

El hecho de que la duración del efecto sea tan corta, hace que sea necesaria la asociación de otras técnicas, como por ejemplo infiltraciones en la región glabelar o en los labios o peeling de la región periorbital (Fig.3) 


\begin{tabular}{|lll|}
\hline AREA & RESULTADO DESEADO & RESULTADO NO DESEADO \\
\hline FRENTE & $\begin{array}{l}\text { Hipocinesia de las fibras mediales del músculo } \\
\text { frontal. } \\
\text { Cinesia normal de las fibras laterlales para } \\
\text { elevar la cola de la ceja. }\end{array}$ & $\begin{array}{l}\text { Hipotonia de las fibras mediales: caída medial } \\
\text { de la ceja y bolsa medial en párpado superior. } \\
\text { Atonia del músculo frontal: pseudoptosis } \\
\text { palpebral. }\end{array}$ \\
\hline GLABELA & Acinesia e hipotonia muscular. & $\begin{array}{l}\text { Atonia: elevación exagerada de la región } \\
\text { medial de las cejas. }\end{array}$ \\
\hline PERIORBITA & $\begin{array}{l}\text { Hipocinesia leve en pacientes hipercinéticos e } \\
\text { hipocinesia moderada en los pacientes } \\
\text { hipertónicos. }\end{array}$ & $\begin{array}{l}\text { Hipotonia o hipocinesia exagerada del } \\
\text { músculo orbicular de los ojos que puede } \\
\text { causar sequedad ocular. }\end{array}$ \\
\hline PERIBUCAL & $\begin{array}{l}\text { Cinesia y coordinación normal entre los } \\
\text { elevadores, depresores y músculo orbicular de } \\
\text { la boca. }\end{array}$ & $\begin{array}{l}\text { Hipotonia o hipocinesia del músculo orbicular } \\
\text { de la boca con parálisis excesiva de la porción } \\
\text { medial del labio superior. }\end{array}$ \\
\hline $\begin{array}{l}\text { SONRISA } \\
\text { GINGIVAL }\end{array}$ & $\begin{array}{l}\text { Hipocinesia del músculo elevador del ala de la } \\
\text { nariz y del labio superior y del músculo } \\
\text { elevador del labio superior. }\end{array}$ & $\begin{array}{l}\text { Atonia de los elevadores y caída del labio } \\
\text { superior. }\end{array}$ \\
\hline CUELLO & $\begin{array}{l}\text { Hipocinesia e hipotonía del músculo platisma } \\
\text { Cuninesia o atonía del músculo platisma: }\end{array}$ \\
\hline
\end{tabular}

Tabla I. Esquema terapeutico para el control de la contracción muscular

\section{¿HIPOCINESIA O ACINESIA? ¿ATONIA O HIPOTONÍA?}

Dependiendo del área de tratamiento, puede ser deseable el bloqueo total de la contracción muscular (acinesia) o la disminución del movimiento de los músculos (hipocinesia).

Para que las estructuras anatómicas permanezcan en la localización adecuada, es de desear que se mantenga un tono muscular mínimos. La hipotonía es aconsejable para las áreas hipertónicas, pero sin embargo debemos temer la atonía, pues puede ser causa de resultados estéticos desastrosos como sucede por ejemplo cuando se produce una seudo-ptosis palpebral bilateral por atonía del músculo frontal. Otro ejemplo sería la aplicación muy baja e inadvertida sobre las arrugas periorbitarias del párpado inferior, que puede causar una atonía del músculo zigomático mayor y la consecuente caída del labio superior (13).

Se aconseja un bloqueo total de la contracción muscular en la región glabelar mediante la parálisis de los músculos corrugadores y procerus. En la región frontal se aconseja un movimiento hipocinético, pues evita la apariencia "congelada" de la cara al mantener algún movimiento. La hipotonía de esta región produce la ptosis de la porción medial de las cejas y la aparición de una bolsa grasa en la región medial del párpado superior.

En la zona de los ojos, si el tratamiento con Toxina Botulínica es excesivo y la musculatura se vuelve hipocinética, habrá una repercusión funcional negativa, con sequedad ocular y edema palpebral inferior. Lo aconsejable para la región periocular es un control de la hipercinesia y de la hipotonía muscular que producen respectivamente el exceso de arrugas en el movimiento y la presencia de arrugas estáticas. En la Tabla I se refleja el comportamiento deseado o no para cada área de tratamiento con Toxina Botulínica.

\section{Conclusiones}

La aplicación de Toxina Botulínica se va mejorando con el paso del tiempo, lográndose un resultado natural fruto de la habilidad del especialista que realiza la inyección a la hora de definir qué áreas deben permanecer con actividad cinética, hipocinética o hipotónica. En la cara, este equilibrio debe ser determinado de acuerdo con el sexo y la edad del paciente. De esta forma, los tratamientos con Toxina Botulínica proporcionarán mejores resultados a los pacientes.

\section{Dirección del autor}

Dr. Mauricio de Maio

Avda. Ibirapuera, 2907 cj 1202

CEP: 04029-200

Sao Paulo. Brasil

e-mail:mauriciodemaio@uol.com.br 


\section{Bibliografía}

1. Maio M.: "The minimal approach: an innovation in facial cosmetic procedures". Aesth Plast Surg.2004 ; 28:295.

2. Lewis CM, Lavell S, Simpson MF: "Patient selection and patient satisfaction“. Clin Plast Surg 1983; 321.

3. Vuyk HD, Zijlker TD.: "Psychosocial aspects of patient counseling and selection: a surgeon's perspective“. Fac Plast Surg. 1995; 11:55.

4. Donofrio L.: "Fat distribution: A morphologic study of the aging face". Dermatol Surg.2000; 26:1107.

5. Barton FE, Gyimesi TM.: "Anatomy of nasolabial fold". Plast Reconstr Surg.1997; 100:1276.

6. Maio, M.: "Envelhecimento". In: Mauricio de Maio: Tratado de Medicina Estética.Vol. 1. 1 ${ }^{a}$ ed., São Paulo, Rocca.2004; Pp 249266.
7. Adamson PA, Kraus WM.: "Management of patient dissatisfaction with cosmetic surgery“. Fac Plast Surg 1995; 11:99.

8. Baker TJ.: "Patient selection and psychological evaluation". Clin Plast Surg 1978, 5:3.

9. Flowers RS., Caputy GG., Flowers SS.: "The biomechanics of brow and frontalis function and its effect on blepharoplasty". Clin Plast Surg.1993; 20:255.

10. Furnas DW.: "The retaining ligaments of the cheek". Plast Reconstr Surg.1989, 83:11.

11. Sarwar D.: "SARWAR D. The "obsessive" cosmetic surgery patient: a consideration of body image dissatisfaction and body dysmorphic disorder“. Plast Surg Nurs 1997; 17:193,209.

12. Katez P.: " The dissatisfied patient". Plast Surg Nurs 1991; 11:13.

13. Lemke BN, Stasior OG.: "The anatomy of eyebrow ptosis". Arch Ophthalmol.1982, 100:981. 


\title{
Toxina botulínica: a relação do tīpo de paciente com a duração de afeito
}

\author{
De Maio, M. *, Ofenböck Magri, I.**, Narvaes Bello, C.*** \\ * Cirugião Plástico e Doutor em Ciências pela Faculdade de Medicina da Universidade de São Paulo \\ * Acadêmica da Faculdade de Medicina do ABC \\ * Hospital das Clínicas. Faculdade de Medicina da Universidade de Medicina de São Paulo
}

Resumo

O uso da toxina botulínica é cada vez mais freqüente em procedimentos estéticos não cirúrgicos. Muitos aspectos, no entanto, ainda necessitam ser esclarecidos. Através do agrupamento de pacientes em diferentes padrões musculares, a duração do efeito pode ser estabelecida e informada aos pacientes.Os grupos consistem em: padrões cinéticos, hipercinéticos e hipertônicos.

O padrão cinético indica que pacientes apresentam uma concordância com a expressão facial e emoções. Eles provavelmente precisarão somente de um tratamento por ano. O padrão hipercinético refere-se a pacientes que não movem seus músculos de acordo com sua mímica. Os músculos faciais contraem rapidamente e a duração varia de 4 a 6 meses. O padrão hipertônico é encontrado naqueles pacientes que são incapazes de relaxar seus músculos faciais e a duração pode ser somente de 2 a 3 meses. O que torna o tratamento frustrante para pacientes e médicos.

Através do entendimento do comportamento muscular, a abordagem do paciente e seu agrupamento de acordo com os específicos padrões musculares possibilita a previsão da duração e orienta sobre os futuros tratamentos.

Palavras chave: Toxina botulínica, Cirugía Cosmética, Padrões musculares.

Introdução

O tratamento estético com toxina botulínica continua sendo uma das terapêuticas não-cirúrgicas mais freqüentes entre todos os procedimentos minimamente invasivos. Técnicas e táticas estão sendo descobertas e implantadas nos terços superior, médio e inferior da face. Apesar do avanço do conhecimento médico, algumas questões ainda continuam com respostas insatisfatórias. Uma das questões cruciais é o tempo de duração do efeito da toxina botulínica e porque há uma variação tão significativa de efeito entre os pacientes (1).

Este capítulo visa fornecer dados para facilitar a abordagem de pacientes e possibilitar a inclusão destes pacientes em grupos de diferentes comportamentos musculares. Visa também informar como orientá-los em relação à duração do efeito e tratamentos futuros $(2,3)$.

\section{COMPORTAMENTO MUSCULAR}

O comportamento muscular se altera com o processo de envelhecimento. A diminuição da acuidade visual, excesso de pele em pálpebra superior e ptose do supercílio resultam da contração exacerbada dos corrugadores e do músculo frontal, respectivamente.

A musculatura da mímica, além de expressar emoções, pode ser útil nos processos compensatórios e adaptativos que ocorrem quando envelhecemos (4). Os músculos levantadores da face tornam-se paulatinamente mais fracos e os abaixadores, pela diminuição da força antagônica, começam a contrair-se promovendo aparência de cansaço e tristeza, como é o caso do abaixador do ângulo da boca $(5,6)$.

Ao observar alguns pacientes se expressando ou conversando, notase que há diferentes padrões de mímica: o paciente "normal", o paciente que se expressa em exagero e aqueles que se expressam de forma contida (5).

\section{HISTORICO}

Quem nunca ouviu algum paciente relatar que não queria ficar com "cara espantada ou paralisada" com uso de toxina botulínica? Logo no início, alguns tratamentos eram realizados de forma excessiva, culmi-

nando em bloqueio muscular indesejável. Como resultado, além do desconforto do paciente, havia o estigma da aplicação da toxina botulínica (7).

A paralisia completa de alguns grupos musculares em pacientes estéticos também passou a confundir a avaliação pelos pacientes e por alguns médicos. O resultado considerado eficiente era bloqueio muscular total, com ausência de movimento. Após 28 dias da aplicação, há o brotamento colateral do axônio terminal e passagem de acetilcolina. Com isso, há inicio de movimento muscular, e, para muitos pacientes, a noção errônea de término de efeito (8).

\section{TIPOS DE PACIENTES \\ Pacientes cinéticos}

Os pacientes cinéticos são os que movem a musculatura da mímica em concordância com as emoções que desejam transmitir. Quando desejam expressar surpresa, elevam os supercílios; a seriedade é transmitida ao contrair os corrugadores. A contração e relaxamento muscular apresentam ritmo agradável ao interlocutor, o qual se sente confortável ao conversar com este tipo de pacientes. A frase de referência destes pacientes é "Eu contraio a musculatura quando expresso emoções" $(9,10)$. Ao exame físico, as linhas ou rugas aparecem somente durante a contração muscular, e, em geral, não são profundas. Não há presença de linhas estáticas nas áreas de movimentação muscular e os músculos apresentam tônus muscular normal.

A duração de efeito em pacientes cinéticos é a maior encontrada entre os diversos tipos de pacientes. Não é incomum que estes pacientes façam a aplicação de toxina botulínica apenas uma vez ao ano; não porque a paralisa muscular dura um ano, porém a movimentação muscular não incomoda o paciente a ponto de realizar mais de uma injeção ao ano.

Para este tipo de pacientes, vale a informação que o efeito pode durar mais do que seis meses e, às vezes, até nove meses.( Fig.1)

\section{Pacientes hipercinéticos}

Os pacientes hipercinéticos contraem a musculatura em ritmo rápido e não necessariamente em concordância com as emoções. O interlocutor tem sensação de inquietude, pois o paciente apresenta contrações da musculatura da mímica em excesso.

Alterna a contração e relaxamento em ritmo acelerado. A frase de referência destes pacientes é "Eu não consigo controlar a mímica facial".

Ao exame físico, as linhas ou rugas estão presentes durante o repouso, mas não são

profundas. Durante a contração muscular, nota-se que a força muscular causa o aprofundamento intenso das linhas estáticas. Em geral, as linhas desaparecem com o afastamento digital e, à palpação, a derme possui espessura uniforme e regular.

Para os pacientes hipercinéticos, a duração é conhecida e estabelecida pela literatura: de quatro a seis meses. Estes pacientes são os únicos que se pode prever facilmente a duração de efeito. Em geral, se a duração se aproximar de seis meses ficam extremamente felizes. Se o efeito durar somente quatro meses, os pacientes costumam se queixar, porém lhes foi avisado anteriormente esta possibilidade. Este tipo de paciente retorna com frequência para as aplicações de toxina botulínica, porque apresenta alta performance de resultado. A presença de movimento muscular já os estimula a realizar tratamentos consecutivos (11) (Fig.2).

\section{Pacientes hipertônicos}

A característica dos pacientes hipertônicos é a dificuldade de relaxar a musculatura da mímica. Em geral, as áreas como glabela, região fron- 


\begin{tabular}{|c|c|c|}
\hline AREA & RESULTADO DESEJADO & RESULTADO INDESEJADO \\
\hline FRONTE & $\begin{array}{l}\text { Hipocinesia nas fibras mediais do músculo } \\
\text { frontal } \\
\text { Cinesia normal das fibras laterais para elevação } \\
\text { da cauda do supercílio }\end{array}$ & $\begin{array}{l}\text { Hipotonia das fibras mediais: queda medial do } \\
\text { supercílio e bolsas mediais em pálpebra superior. } \\
\text { Atonia do músculo frontal: pseudo-ptose } \\
\text { palpebral. }\end{array}$ \\
\hline GLABELA & Acinesia e hipotonia muscular & $\begin{array}{l}\text { Atonia: elevação exacerbada da região medial } \\
\text { dos supercílios. }\end{array}$ \\
\hline PERIORBITAL & $\begin{array}{l}\text { Hipocinesia leve em pacientes hipercinéticos } \\
\text { e hipocinesia moderada nos pacientes } \\
\text { hipertônicos }\end{array}$ & $\begin{array}{l}\text { Hipotonia ou hipocinesia exagerada do } \\
\text { músculo orbicular dos olhos que pode causar } \\
\text { ressecamento ocular }\end{array}$ \\
\hline PERIBUCAL & $\begin{array}{l}\text { Cinesia e coordenação normal entre os } \\
\text { levantadores, abaixadores e músculo } \\
\text { orbicular da boca. }\end{array}$ & $\begin{array}{l}\text { Hipotonia ou hipocinesia do músculo orbicular } \\
\text { da boca com paralisia excessiva da porção } \\
\text { medial do lábio superior. }\end{array}$ \\
\hline $\begin{array}{l}\text { SORRISO } \\
\text { GENGIVAL }\end{array}$ & $\begin{array}{l}\text { Hipocinesia do músculo levantador da asa do } \\
\text { nariz e do lábio superior e do músculo } \\
\text { levantador do lábio superior }\end{array}$ & $\begin{array}{l}\text { Atonia dos levantadores e queda do lábio supe- } \\
\text { rior }\end{array}$ \\
\hline CERVICAL & Hipocinesia e hipotonia do músculo platisma & $\begin{array}{l}\text { Acinesia ou atonia do músculo platisma: } \\
\text { problemas de deglutição }\end{array}$ \\
\hline
\end{tabular}

Tabela I : Esquema terapêutico para o padrão de contração muscular

tal e comissura oral apresentam rugas profundas tanto em repouso quanto em movimento.

Ao exame físico, quando se solicita ao paciente para realizar movimentos de contração e relaxamento da musculatura da mímica, nota-se que há incapacidade de relaxamento total da musculatura, que permanece com variável grau de contração. À palpação, nota-se que há irregularidade na pele com grande diminuição de derme ao longo da ruga.

Este é o grupo de pacientes mais complicados de abordar no que diz respeito à duração. Pelo fato da musculatura apresentar-se hipertônica, devem as primeiras aplicações objetivar, o bloqueio muscular e conseqüiente relaxamento da região tratada e não desaparecimento completo da ruga. Portanto, este tipo de paciente deve ser avisado que a duração pode ser de dois a três meses. É claro que haverá desapontamento, porém é sempre melhor que o paciente seja previamente avisado (12).

O fato da duração de efeito ser tão curta faz com que seja necessária a associação com outras técnicas, como por exemplo, o preenchimento na região glabelar e lábios ou peelings na região periorbital.( Fig.3)

\section{HIPOCINESIA OU ACINESIA? ATONIA OU HIPOTONIA?}

Dependendo da área de tratamento, pode se desejar que haja bloqueio total da contração muscular (acinesia) ou apenas diminuição da movimentação muscular, conhecida como hipocinesia.

Para que as estruturas anatômicas permaneçam nos locais adequados, é desejável que se mantenha o tônus muscular mínimo. A hipotonia é desejada nas áreas hipertônicas, mas a atonia é temida, pois causa resultados estéticos desastrosos, como por exemplo, a pseudo-ptose palpebral bilateral por atonia do músculo frontal. Um outro exemplo é a aplicação muito baixa e inadvertida das rugas periorbitais da pálpebra inferior que pode causar a atonia do músculo zigomático maior e conseqüente queda do lábio superior (13).
O bloqueio total da contração muscular é desejado na região glabelar pela paralisia dos corrugadores e prócero. A movimentação hipocinética é desejada na região frontal, pois evita a aparência congelada da face, por manter algum movimento. A hipotonia desta região resulta em ptose da porção medial dos supercílios bem como aparecimento de bolsa de gordura na região medial da pálpebra superior.

Na região dos olhos, se o tratamento com toxina botulínica for excessivo, tornando a musculatura hipocinética, haverá repercussão funcional negativa, como ressecamento ocular ou edema em pálpebra inferior. O que se deseja na região periocular é o controle da hipercinesia ou hipertonia muscular que causam respectivamente excesso de ruga à movimentação ou presença de rugas estáticas, respectivamente. Na Tabela I, pode-se visualizar o comportamento desejado e indesejado por área com tratamento de toxina botulínica tipo A.

\section{Conclusão}

A aplicação de toxina botulínica vem se aprimorando com o passar do tempo e o resultado natural resulta da habilidade do injetor em definir quais áreas devem permanecer com comportamento cinético, hipocinético ou hipotônico. Na face, este equilíbrio deve ser determinado de acordo com o sexo e idade do paciente. Desta forma, os tratamentos com toxina botulínica poderão proporcionar melhor satisfação aos pacientes. 